\title{
Herpes Zoster May Be a Marker for COVID-19 Infection During Pregnancy
}

\author{
Mohamed L. Elsaie, MD; Eman A.Youssef, MD; Hesham A. Nada, MD
}

\section{PRACTICE POINTS}

- The vesicular rash of coronavirus disease 2019 (COVID-19) has been reported to have different forms of presentation.

- Pregnant women appear to be at increased risk for complications from COVID-19 infection.

- The clinical presentation of herpes zoster should be carefully monitored and reported for further assessment, especially if associated with other signs of COVID-19 infection.
Varicella-zoster virus infection causes 2 distinct forms of disease: varicella (commonly known as chickenpox) and herpes zoster $(\mathrm{HZ})($ commonly known as shingles). Primary varicella-zoster virus infection results in the diffuse vesicular rash that is characteristic of chickenpox. Following primary infection, varicella-zoster virus remains dormant in the dorsal root ganglia. This latent phase usually lasts for several decades before reactivation occurs. Varicella-zoster virus reactivation normally presents as $\mathrm{HZ}$ in middle-aged adults. A number of cutaneous skin manifestations have appeared in relation to the newly diagnosed coronavirus disease 2019 (COVID-19) pandemic and continue to emerge every day. We report a case of $\mathrm{HZ}$ complication in a COVID-19-positive woman who was 27 weeks pregnant.

Cutis. 2020;106:318-320.

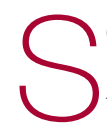
evere acute respiratory syndrome coronavirus 2 (SARS-CoV-2) is the most recently identified member of the zoonotic pathogens of coronaviruses.
It caused an outbreak of pneumonia in December 2019 in Wuhan, China. ${ }^{1}$ Among all related acute respiratory syndromes (SARS-CoV, Middle East respiratory syndrome coronavirus), SARS-CoV-2 remains to be the most infectious, has the highest potential for human transmission, and can eventually result in acute respiratory distress syndrome. ${ }^{2,3}$

Only 15\% of coronavirus disease 2019 (COVID-19) cases progress to pneumonia, and approximately $5 \%$ of these cases develop acute respiratory distress syndrome, septic shock, and/or multiple organ failure. The majority of cases only exhibit mild to moderate symptoms., ${ }^{4,5} \mathrm{~A}$ wide array of skin manifestations in COVID-19 infection have been reported, including maculopapular eruptions, morbilliform rashes, urticaria, chickenpoxlike lesions, livedo reticularis, COVID toes, erythema multiforme, pityriasis rosea, and several other patterns. ${ }^{6}$ We report a case of herpes zoster (HZ) complication in a COVID-19positive woman who was 27 weeks pregnant.

\section{Case Report}

A 36-year-old woman who was 27 weeks pregnant was referred by her obstetrician to the dermatology clinic. She presented with a low-grade fever and a vesicular painful rash. Physical examination revealed painful, itchy, dysesthetic papules and vesicles on the left side of the forehead along with mild edema of the left upper eyelid but no watering of the eye or photophobia. She reported episodes of fever (temperature, $38.9^{\circ} \mathrm{C}$ ), fatigue, and myalgia over the last week. She had bouts of dyspnea and tachycardia that she thought were related to being in the late second trimester of pregnancy. The area surrounding

Dr. Elsaie is from the Department of Dermatology, National Research Centre, Giza, Egypt, and the Miller School of Medicine, University of Miami,

Florida. Dr. Youssef is from the Department of Clinical and Chemical Pathology, Cairo University, Egypt. Dr. Nada is from the Department of Dermatology, Venereology, and Anderology, Faculty of Medicine, Suez Canal University, Ismailia, Egypt.

The authors report no conflict of interest.

Correspondence: Mohamed L. Elsaie, MD (Egydoc77@yahoo.com).

doi: $10.12788 /$ cutis.0133 


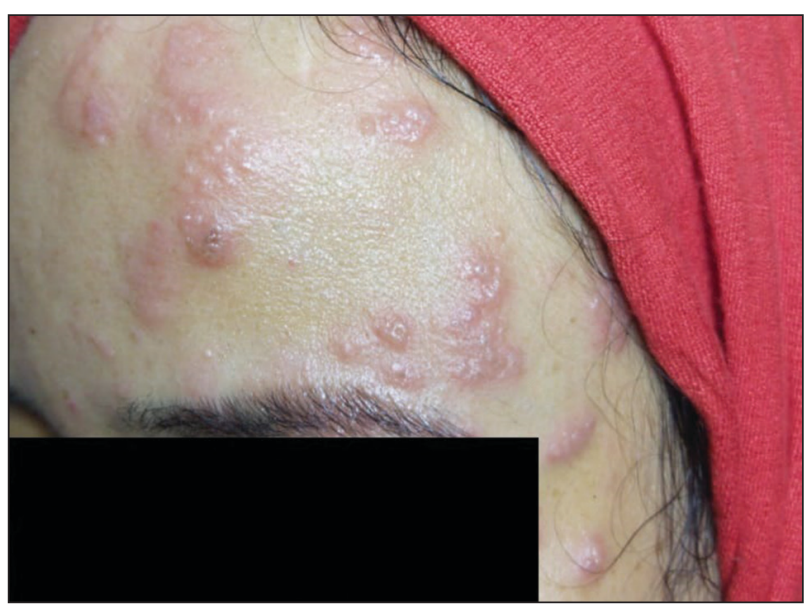

Herpes zoster presentation of coronavirus disease 2019. Multiple blisters and vesicles on the forehead of a pregnant woman.

the vesicular eruption was tender to touch. No dry cough or any gastrointestinal or urinary tract symptoms were noted. She reported a burning sensation when splashing water on the face or when exposed to air currents. One week following the initial symptoms, she experienced a painful vesicular rash along the upper left forehead (Figure) associated with eyelid edema. Oral and ocular mucosae were free of any presentations. She had no relevant history and had not experienced any complications during pregnancy. A diagnosis of $\mathrm{HZ}$ was made, and she was prescribed valacyclovir $1 \mathrm{~g}$ 3 times daily for 7 days, acetaminophen for the fever, and calamine lotion. We recommended COVID-19 testing based on her symptoms. A chest radiograph and a positive nasopharyngeal smear were consistent with COVID-19 infection. She reported via telephone follow-up 1 week after presentation that her skin condition had improved following the treatment course and that the vesicles eventually dried, leaving a crusting appearance after 5 to 7 days. Regarding her SARS-CoV-2 condition, her oxygen saturation was $95 \%$ at presentation; she self-quarantined at home; and she was treated with oseltamivir $75 \mathrm{mg}$ orally every 12 hours for 5 days, azithromycin $500 \mathrm{mg}$ orally daily, acetaminophen, and vitamin $\mathrm{C}$. Electronic fetal heart rate monitoring and ultrasound examinations were performed to assess the condition of the fetus and were reported normal. At the time of writing this article, she was 32 weeks pregnant and tested negative to 2 consecutive nasopharyngeal swabs for COVID-19 and was in good general condition. She continued her pregnancy according to her obstetrician's recommendations.

\section{Comment}

The incubation time of COVID-19 can be up to 14 days. Fever, dry cough, fatigue, and diarrhea have been speculated to be clinical symptoms; however, many cases may be asymptomatic. Aside from a medical or travel history at risk for COVID-19, diagnosis can be confirmed by detection of viral RNA by reverse transcriptasepolymerase chain reaction for nasopharyngeal swabs or bronchoalveolar fluid. Patients who are immunocompromised, older, or male or who have a history of cardiovascular conditions or debilitating chronic conditions are at an increased risk for severe disease and poor outcome compared to younger healthy individuals. ${ }^{7}$

The vesicular rash of COVID-19 has been reported to have different forms of presentation. A diffuse widespread pattern resembling hand-foot-and-mouth disease and a localized monomorphic pattern resembling chickenpox but with predilection to the trunk has been described. ${ }^{8}$

Physiologic changes in the immune and cardiopulmonary systems during pregnancy (eg, diaphragm elevation, increased oxygen consumption, edema of the respiratory tract mucosae) make pregnant women intolerant to hypoxia. The mortality rate of the 1918 influenza pandemic was $2.6 \%$ in the overall population but $37 \%$ among pregnant women. ${ }^{9}$ In 2009 , pregnant women were reported to be at an increased risk for complications from the H1N1 influenza virus pandemic, with a higher estimated rate of hospital admission than the general population. ${ }^{10}$ In 2003, approximately $50 \%$ of pregnant women who received a diagnosis of SARS-CoV were admitted to the intensive care unit, approximately $33 \%$ of pregnant women with SARS-CoV required mechanical ventilation, and the mortality rate was as high as $25 \%$ for these women. ${ }^{11}$ To date, data on the effects of COVID-19 in pregnancy are limited to small case series. ${ }^{12-15}$

It was confirmed that COVID-19 infection is accompanied by a reduction in lymphocytes, monocytes, and eosinophils, along with a notable reduction of CD4/CD8 T cells, B cells, and natural killer cells. It was further revealed that nonsurvivor COVID-19 patients continued to show a decrease in lymphocyte counts along the course of their disease until death. ${ }^{16-18}$

Different mechanisms for lymphocyte depletion and deficiency were speculated among COVID-19 patients and include direct lymphocyte death through coronavirus angiotensin-converting enzyme 2-lymphocyte-expressed receptors; direct damage to lymphatic organs, such as the thymus and spleen, but this theory needs to be further investigated; direct lymphocyte apoptosis mediated by tumor necrosis factor $\alpha$, IL-6, and other proinflammatory cytokines; and direct inhibition of lymphocytes by metabolic upset, such as acidosis..$^{19,20}$

These causes may precipitate lymphopenia and impaired antiviral responses. ${ }^{21}$ It also has been postulated that the functional damage of $\mathrm{CD} 4^{+} \mathrm{T}$ cells may predispose patients with COVID-19 to severe disease. ${ }^{22}$ Such immune changes can render a patient more susceptible to developing shingles by reactivating varicella-zoster virus, which could be a sign of undiagnosed COVID-19 infection in younger age groups.

Two earlier reports discussed HZ among COVID-19-diagnosed patients. Shors ${ }^{23}$ presented a case of a patient who developed varicella-zoster virus reactivation 
of the V2 dermatome during the course of COVID-19 infection. In addition, the patient developed severe acute herpetic neuralgia despite the early initiation of antiviral therapy. ${ }^{23}$ Elsaie et $\mathrm{al}^{24}$ described 2 cases of patients during the pandemic who first presented with $\mathrm{HZ}$ before later being diagnosed with COVID-19 infection.

New information and cutaneous manifestations possibly related to COVID-19 are emerging every day. We report a pregnant female presenting with $\mathrm{HZ}$ during the course of COVID-19 infection, which suggests that the clinical presentation of $\mathrm{HZ}$ at the time of the current pandemic, especially if associated with other signs of COVID-19 infection, should be carefully monitored and reported for further assessment.

Acknowledgment-The authors would like to thank all the health care workers who have been fighting COVID-19 in Egypt and worldwide.

\section{REFERENCES}

1. Li Q, Guan X, Wu P, et al. Early transmission dynamics in Wuhan, China, of novel coronavirus-infected pneumonia. $N$ Engl J Med. 2020;382:1199-1207.

2. Zhang $\mathrm{YZ}$, Holes EC. A genomic perspective on the origin and emergence of sars-cov-2. Cell. 2020;181:223-227.

3. Prompetchara E, Ketloy C, Palaga T. Immune responses in COVID-19 and potential vaccines: lessons learned from SARS and MERS epidemic. Asian Pac J Allergy Immunol. 2020;38:1-9.

4. Huang C, Wang Y, Li X, et al. Clinical features of patients infected with 2019 novel coronavirus in Wuhan0, China. Lancet. 2020;395:497-506.

5. Xu Z, Shi L, Wang Y, et al. Pathological findings of COVID-19 associated with acute respiratory distress syndrome. Lancet Respir Med. 2020; 8:420-422.

6. Wollina U, Karadağ AS, Rowland-Payne C, et al. Cutaneous signs in COVID-19 patients: a review. Dermatol Ther. 2020;33:e13549.

7. Lauer SA, Grantz KH, Bi Q, et al. The incubation period of coronavirus disease 2019 (COVID-19) from publicly reported confirmed cases: estimation and application. Ann Intern Med. 2020;172:577-582.

8. Fernandez-Nieto D, Ortega-Quijano D, Jimenez-Cauhe J, et al. Clinical and histological characterization of vesicular COVID-19 rashes: a prospective study in a tertiary care hospital. Clin Exp Dermatol. 2020;45:872-875.
9. Gottfredsson M. The Spanish flu in Iceland 1918. Lessons in medicine and history [in Icelandic]. Laeknabladid. 2008;94:737-745.

10. Jamieson D, Honein M, Rasmussen S, et al. H1N1 2009 influenza virus infection during pregnancy in the USA. Lancet. 2009;374:451-458.

11. Ksiazek TG, Erdman D, Goldsmith CS. A novel coronavirus associated with severe acute respiratory syndrome. N Engl J Med. 2003; 348:1953-1966.

12. Chen H, Guo J, Wang C, et al. Clinical characteristics and intrauterine vertical transmission potential of COVID-19 infection in nine pregnant women: a retrospective review of medical records. Lancet. 2020;395:809-815.

13. Zhu H, Wang L, Fang C, et al. Clinical analysis of 10 neonates born to mothers with 2019-nCov pneumonia. Transl Pediatr. 2020;9:51-60.

14. Liu Y, Chen H, Tang K, et al. Clinical manifestations and outcome of SARS-CoV-2 infection during pregnancy [published online March 4, 2020]. J Infect. doi:10.1016/j.jinf.2020.02.028.

15. Zhang L, Jiang Y, Wei M, et al. Analysis of the pregnancy outcomes in pregnant women with COVID-19 in Hubei Province [in Chinese]. Zhonghua Fu Chan Ke Za Zhi. 2020;55:166-171.

16. Henry BM, de Oliveira MHS, Benoit S, et al. Hematologic, biochemical and immune biomarker abnormalities associated with severe illness and mortality in coronavirus disease 2019 (COVID-19): a metaanalysis. Clin Chem Lab Med. 2020;58:1021-1028.

17. Cai Q, Huang D, Ou P, et al. COVID-19 in a designated infectious diseases hospital outside Hubei Province, China. Allergy. 2020; 75:1742-1752.

18. Ruan Q, Yang K, Wang W, et al. Clinical predictors of mortality due to COVID-19 based on an analysis of data of 150 patients from Wuhan, China. Intensive Care Med. 2020;46:846-884

19. Kumar A, Anil A, Sharma P, et al. Clinical features of COVID-19 and factors associated with severe clinical course: a systematic review and meta-analysis [preprint]. SSRN. doi:10.2139/ssrn.3566166.

20. Xu H, Zhong L, Deng J, et al. High expression of ACE2 receptor of 2019-nCoV on the epithelial cells of oral mucosa. Int J Oral Sci. 2020;12. https://doi.org/10.1038/s41368-020-0074-x.

21. Li H, Liu L, Zhang D, et al. SARS-CoV-2 and viral sepsis: observations and hypotheses. Lancet. 2020;395:1517-1520.

22. Zheng M, Gao Y, Wang G, et al. Functional exhaustion of antiviral lymphocytes in COVID-19 patients. Cell Mol Immunol. 2020; 17:533-535.

23. Shors AR. Herpes zoster and severe acute herpetic neuralgia as a complication of COVID-19 infection. JAAD Case Rep. 2020;6:656-657.

24. Elsaie ML, Youssef EA, Nada HA. Herpes zoster might be an indicator for latent COVID 19 infection [published online May 23, 2020]. Dermatol Ther. doi:10.1111/dth.13666. 